\title{
Aldesleukin Prodrug NKTR-214
}

National Cancer Institute

\section{Source}

National Cancer Institute. Aldesleukin Prodrug NKTR-214. NCI Thesaurus. Code C131019.

A recombinant form of the endogenous cytokine interleukin-2 (IL-2) conjug ated to six releasable polyethylene glycol (PEG) chains, with potential immunostimulating activity. Upon administration of the aldesleukin prodrug NKTR-214, the IL-2 moiety binds to the IL-2 receptor beta subunit (IL2Rb; IL2Rbeta; CD122). The binding of IL-2 to IL2Rb activates IL2Rb-mediated signaling, which activates cytotoxic T-lymphocytes (CT LS) and natural killer (NK) cells, and induces expression of certain cytotoxic cytokines, such as interferon-gamma (IFNg) and transforming growth factor-beta (TGFb). The specific induction of T-cell-mediated cytotoxic immune responses against tumor cells primarily causes tumor cell destruction. IL2Rb plays a key role in the proliferation and activation of effector T-cells. PEG conjug ation prevents IL-2 binding to the IL2Ralpha subunit (IL2Ra) because signaling through IL2Ra activates CD4-positive regulatory, immunosuppressive T-cells (Tregs), which would suppress tumor cell killing. 